(FWHM), at a position $93 \mathrm{~cm}$ downstream from the CRL using 6.5-keV photons. The spot diameter was larger than expected, which was attributed to surface irregularities or the uncertainty of the source.

The Gaussian transmission profile FWHM of the Be CRL was also measured by scanning a $25 \mu \mathrm{m} \times 25 \mu \mathrm{m}$ beam across the CRL and was determined to be $321 \mu \mathrm{m}$ with on-axis transmission of $9 \%$ at $6.5 \mathrm{keV}$ photon energy. The attenuation aperture was thus calculated to be $607 \mu \mathrm{m}$. Consequently, using the experimental data, a gain of 1.5 was calculated for this lens, compared with a theoretical value of 6 . The obtainable gain is dependent on the source size used for the experiments, and much higher gains would be expected from thirdgeneration and beyond $x$-ray facilities.

According to the researchers, the use of CRLs made of beryllium "can achieve submeter focal lengths at lower x-ray energies $(\sim 6.5 \mathrm{keV})$ than for previously reported CRLs and still have what are believed to be the largest reported apertures $(\sim 600 \mu \mathrm{m})$. Thus, it is expected that Be CRLs can outperform lenses constructed of higher-atomic-number materials at energies below $30 \mathrm{keV}$."

Calin Miclaus

\section{Heating Allows Birefringence Tuning in Microstructured Optical Fibers Partially Filled with a Polymer}

Recently, researchers have shown that microstructured photonic-crystal optical fibers (MOFs) formed by incorporating air holes running along the length of the fiber can enable additional control of birefringence, dispersion, and nonlinearity of the fibers. Among other novel techniques, introducing active materials into the air holes of microstructured optical fibers (MOFs) improves the capabilities of the devices fabricated. As they demonstrate in their article in the May 15 issue of Optics Letters, C. Kerbage, B.J. Eggleton, and coworkers from OFS Fitel Laboratories (now OFS Laboratories) in New Jersey, were able to induce and tune birefringence in MOFs.

The researchers chose MOFs comprised of a central germanium-doped core, $8 \mu \mathrm{m}$ in diameter, encircled symmetrically by six air holes. They fabricated an all-fiber polarization controller, $125 \mu \mathrm{m}$ in diameter but tapered along $1 \mathrm{~cm}$ of the length down to $\sim 30 \mu \mathrm{m}$ in diameter. This was to ensure that the mode to guide light was by total internal reflection at the silica-airhole interface. The fiber was tapered adiabatically to achieve low intrinsic loss. Since MOFs with a rotational symmetry larger than twofold are not birefringent, the scientists had to break the sixfold symmetry by filling two opposite air holes with an acrylate-based polymer. The monomer filling, limited to the tapered area, was fed at $0.01 \mathrm{~cm} / \mathrm{s}$ and cured using UV rays for $15 \mathrm{~min}$. The resulting polymer had a refractive index of 1.434 at ambient temperature and at a wavelength of $1550 \mathrm{~nm}$, although it is temperature-dependent $\left(\mathrm{d} n / \mathrm{d} T \sim-4 \times 10^{-4}{ }^{\circ} \mathrm{C}^{-1}\right)$, and hence is controlled by heating. After placing the device in a capillary heater, the research team applied a 1550-nm polarized laser beam to the fiber to yield an output light beam, which was analyzed for its polarization properties and converted into the Stokes parameters. Results after heating increments were expressed as a rotation of the Stokes vector, and all results were plotted on the Poincaré sphere.

The beam propagation method (BPM) simulated the distortion in the mode fields of this structure. Calculations performed using BPM were close to the experimental results. When the index is lower than that of silica, the light is guided by total internal reflection, and birefringence is low. When the infused polymer has an index near to that of silica, the mode of reflection becomes more asymmetric, and birefringence increases. Analysis of the polarization measurements using the Poincaré sphere show three rotations $(6 \pi)$ along the heating range of the capillary heater. Also, as the researchers reported, the calculated birefringence change reached $\sim 4.4 \times 10^{-4}$, and the tuning coefficient was $\sim 15 \mathrm{rad} / \mathrm{m}^{\circ} \mathrm{C}$. Hysteresis effects were negligible.

$$
\text { SIARI S. SOSA }
$$

\section{Osmium Exhibits Lowest Experimentally Determined Compressibility}

A group of researchers at Lawrence Livermore National Laboratory have determined through experiment and theoretical calculations that the element osmium (Os) has a lower compressibility (or higher bulk modulus $B_{0}$, the reciprocal of compressibility) than diamond, the hardest and least compressible material known to date. As reported by $\mathrm{H}$. Cynn and coworkers in the April 1 issue of Physical Review Letters, the experimentally determined value of $B_{0}$ for osmium is $462 \mathrm{GPa}$, compared with $443 \mathrm{GPa}$ for diamond. Artificially quenched metastable phases of $\mathrm{ZrO}_{2}$ and $\mathrm{TiO}_{2}$ have been reported to have bulk moduli close to that of diamond at best. A simple chemical bonding model and estimates based on the cohesive energy have suggested that Os should have a large $B_{0}$. In addition, Os has the highest Vickers hardness among the $5 d$ transition metals.
Using synchrotron x-ray sources at the National Synchrotron Light Source (Brookhaven National Laboratory) and the Stanford Synchrotron Radiation Laboratory (Stanford University), diamond-anvil cell-compression studies were carried out for three transition metals: ruthenium $(\mathrm{Ru})$, iridium (Ir), and osmium. Condensed Ar was used as a pressure medium. Pressure information was determined using luminescence from ruby grains (grain size $<3 \mu \mathrm{m}$ ). Powder samples of $\mathrm{Ru}$, Ir, and Os (grain size $<5 \mu \mathrm{m}$ ) were packed into a small hole drilled into a rhenium gasket. Synchrotron x-ray diffraction measurements yielded lattice compressions.

A plot of the reduced volume as a function of pressure using the Birch-Murnaghan equation of state (EOS) fits for the three test materials, as well as for tungsten and diamond, shows that Os exhibits the least contraction as the external pressure is increased. The Holzapfel and Vinet EOS models were also applied to the experimental data to ensure consistency of the results for $B_{0}$. The three fitting algorithms all yielded identical results, in order of decreasing compressibility: Ru, Ir, diamond, Os. Theoretical estimates of $B_{0}$ were obtained using a full-potential linear muffin-tin orbital method to calculate the EOS. The calculated values of $B_{0}$ for the $5 d$ transition metals show the same trend as the experiments.

According to the research team, this result opens new possibilities in the search for superhard materials among transitionmetal compounds and alloys.

JUNE LAU

\section{Nanoparticle Mediates Improvement in $\mathrm{Si}$ Electroluminescence Efficiency}

Researchers at National Taiwan University have obtained a several-ordersof-magnitude electroluminescence (EL) efficiency increase from a metal oxide semiconductor (MOS) tunneling diode by replacing the oxide layer within the device with a layer of $\mathrm{SiO}_{2}$ nanoparticles (NPs). C.F. Lin and co-workers have determined that the improved EL is the result of an increase in the density of radiative recombination states in the diode that stems from the spatial confinement of the charge carriers in the nanoparticles. The EL efficiency improvement allows for the use of $\mathrm{Si}$ in optoelectronic devices.

As described in the May 1 issue of Optics Letters, the researchers prepared the tunneling diodes to study the improvement by first removing the oxide layer from Si wafers. This was followed by spincoating 12-nm $\mathrm{SiO}_{2} \mathrm{NPs}$ onto the wafers. $\mathrm{Al}$ was evaporated on top of the NP layer 
and on the bottom of the Si wafer. Afterwards, Au wire leads were attached to both Al layers with Ag paint.

The current-voltage characteristics of over 100 diodes were measured. The EL of the devices was found to arise from the $\mathrm{Si}$ only. The efficiency of the EL varied between $10^{-6}$ and $10^{-4}$ at an injection current of $50 \mathrm{~mA}$. These efficiency values are several orders of magnitude higher than those previously observed for MOS diodes. The rise and fall times of the diodes varied from $4.5 \mu$ s to $20 \mu \mathrm{s}$, and $1.5 \mu$ s to $15 \mu \mathrm{s}$, respectively. Longer rise times were found to correspond to longer fall times and improved EL efficiencies. The researchers attributed the variations in the measured parameters to variations in the nonradiative recombination process in the devices. In devices with fewer nonradiative recombination, the rise and fall times are longer, and the fraction of recombination due to the radiative recombination process is larger. The researchers speculate that the variation in the radiative recombination state densities arise from variations in silicon quality across the wafer, with the recombination being most efficient for high-quality spots.

The devices with larger rise and fall times also exhibited near-lasing-threshold behavior - a rapid output power increase at a threshold value of the input current. Threshold behavior was accompanied by the appearance of resonance modes in the EL spectrum. The researchers attribute the near-lasing behavior to the improved luminescence efficiency coupled to the low-absorption cross section of the $\mathrm{Si}$ and the formation of a quasi-cavity by two reflective metal layers in the device.

GREG KHITROV

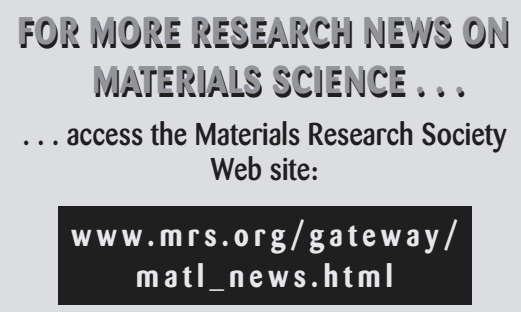

\section{News of MRS Members/Materials Researchers}

\section{Robert Langer Receives the $\mathbf{2 0 0 2}$ Charles Stark Draper Prize}

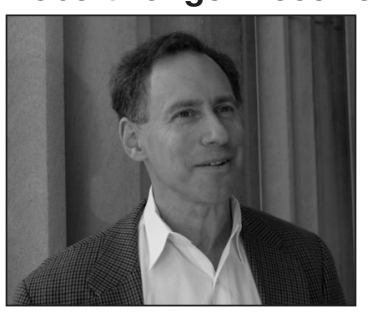

Robert Langer, Kenneth J. Germeshausen Professor of Chemical and Biomedical Engineering at the Massachusetts Institute of Technology, has been awarded the Charles Stark Draper Prize for his invention of medical technologies that help prolong lives and ease suffering. Credited with developing biocompatible polymer technologies that control the release of medicine over timefrom weeks to years-Langer's contributions have significantly advanced the controlled drug-delivery industry.

His engineering of polymer plastics is now allowing delivery of medicine in unique ways to difficult locations in the body. One of his biodegradable polymer inventions to treat brain cancer was the first such chemotherapy that could be delivered directly to a tumor site.

Considered to be both a prolific innovator and researcher, Langer's patents have been licensed or sublicensed to over 80 pharmaceutical, chemical, biotechnology, and medicaldevice companies; a number of companies were launched on the basis of these patent licenses. He nurtures his students into taking their thesis work to the marketplace, therefore making him an example of the role academic research engineers can play in bridging academia and industry.

Langer has written 700 articles. He has also written 400 patents, one of which was cited as the outstanding patent in Massachusetts in 1988 and one of the 20 outstanding patents in the United States.

Langer has received more than 80 major awards, including the Gairdner Foundation International Award in 1996, and the Lemelson-MIT Prize in 1998. In 1989, Langer was elected to the Institute of Medicine, and in 1992 he was elected to both the National Academy of Engineering and the National Academy of Sciences.

He has served, at various times, on eight boards of directors and 20 scientific advisory boards for such companies as Alkermes, Mitsubishi Pharmaceuticals, Warner-Lambert, and Guilford Pharmaceuticals.

Langer received his bachelor's degree from Cornell University in 1970 and his doctorate from MIT in 1974, both in chemical engineering. He has received honorary doctorates from the Swiss Federal Institute of Technology Zurich (ETH Zurich); the Technion-Israel Institute of Technology; and the Université Catholique de Louvain, Belgium. He is chair of the U.S. Food and Drug Administration's Science Board, the administration's highest advisory board.

The Draper Prize is awarded annually and consists of $\$ 500,000$ and a gold medallion. The prize was established by the National Academy of Engineering (NAE) with an $\$ 8$ million endowment from the Charles Stark Draper Laboratory, to honor the "father of inertial navigation." It is awarded for innovative engineering achievement or a body of work extending over a period of years. The work must demonstrate a proven innovation that contributes to human welfare and freedom. NAE is an independent, nonprofit institution.
Andrzej Deptula and Wiestawa Lada (Institute of Nuclear Chemistry and Technology, Warsaw) received two awards from the INNOVATION 2001 International Exhibition of Inventions held on October 17-19, 2001, in Gdansk, Poland: First Award and the Cup of the Rector Navy Academy for "Method for Preparation of High-Temperature Superconductors," invented with T. Olczak, M.T. Lanagan, S.E. Dorris, K.C. Goretta, and R.B. Peoppel; and First Award and Gold Medal for "Method for Preparing of Calcium Phosphates Layers, Especially Hydroxyapatite," invented with R.Z. LeGeros, T. Olczak, and J.P. LeGeros.

Brij M. Moudgil of the University of Florida has been selected to receive the institution's Research Foundation Professorship award in recognition of his distinguished record of research and scholarship.

Rustum Roy of The Pennsylvania State University has been honored by the Ministry of Education, Culture, Sports, and Technology of Japan for exceptionally meritorious service to science in Japan, in a ceremony at the ministry presided over by Minister Atsuko Toyama on March 19, 2002. This award recognizes the contributions that Roy has made over a 35-year period to science and technology in Japan.

S.K. Sundaram of Pacific Northwest National Laboratory (PNNL) has been honored as an "Outstanding Mentor" under the Office of Science, Fellowship Programs, of the Department of Energy. He has also received the PNNL Director's 2002 Fitzner-Eberhardt award for outstanding contributions to science and engineering education. 